Recepción: 17 / 02 / 2017

Aceptación: 13 / 04 / 2018

Publicación: 08 / 05 / 2018

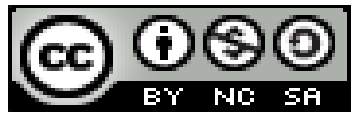

Ciencias de la educación

Artículo de Revisión

\title{
Formación de valores en la Educación Superior
}

\author{
Formation of values in higher education
}

Formación de valores en la educación superior

\author{
Lucia G. Chávez-Ruano ${ }^{\mathrm{I}}$ \\ luciachavez@gmail.com \\ Alfredo N. Tenorio-Obregón II \\ tenorioo86@yahoo.es
}

Correspondencia: luciachavez@gmail.com

\begin{abstract}
I Licenciado en Ciencias de la Educación Especialidad: Física y Matemáticas, Profesor De Especialidad: Física y Matemáticas, Magister en Desarrollo del Pensamiento y la Educación, Docente de la Universidad "Luis Vargas Torres” Esmeraldas, Ecuador.

II Master en Ciencias Especialidad Docencia Universitaria, Doctor en Ciencias de la Educación Mención Investigación y Planificación Educativa, Licenciado en Ciencias de la Educación Profesor de Enseñanza Secundaria en la Especialización de Química y Biología, Docente de la Universidad "Luis Vargas Torres" Esmeraldas, Ecuador.
\end{abstract}




\section{Resumen}

La presente investigación trata sobre la formación de valores en la educación superior, educar en valores es ayudar al individuo a fin de que tome las decisiones correctas en circunstancias conflictivas, cada día la sociedad demanda profesionales competentes, para nadie es un secreto la crisis de valores que viven los profesionales universitarios, es de vital importancia que los docentes estén preparados bajo el enfoque de valores, por lo tanto la misión que todo docente debe tener es convertirse en el modelo del alumno para que este aprenda a imitarlo, pues es esta la manera más eficaz de enseñar valores a los alumnos, por otra parte los docentes deben tener claro que no solo están formando buenos profesionales desde el punto de vista del conocimiento, sino que también están formando seres humanos que impactaran en la solución de los problemas de una sociedad. La educación en valores no solo depende de los profesores es un trabajo en conjunto con las autoridades universitarias, en este trabajo vemos como la Universidad "Luis Vargas Torres" de Esmeraldas, Ecuador, hizo un estudio que tuvo como finalidad recoger sugerencias en cuanto a la formación de valores de las Carreras de Ingeniería, es por ello las universidades deben estar alertas implementando herramientas que permitan detectar las debilidades en cuanto a los programas de formación universitaria.

Palabras clave: Valores; universidad; docentes; formación universitaria.

\section{Abstract}

The present research deals with the formation of values in higher education, educating in values is helping the individual in order to make the right decisions in conflicting circumstances, every day society demands competent professionals, for nobody is a secret the crisis of values that university professionals live, it is vital that teachers are prepared under the values approach, therefore the mission that every teacher should have is to become the model of the student so that he learns to imitate him, for this is the way more effective to teach values to students, on the other hand teachers must be clear that not only are they forming good professionals from the point of view of knowledge, but also are forming human beings that impact on solving the problems of a society . Education in values not only depends on the teachers, it is a work in conjunction with the university authorities, in this work we see how the University "Luis Vargas Torres" of Esmeraldas, Ecuador, made a study that had as a purpose to collect suggestions as to the training 
of values of the Engineering Careers, this is why the universities must be alert to implement tools that allow to detect the weaknesses in the programs of university formation.

Keywords: Values; university; teachers; university education.

\section{Resumo}

A investigação sobre a formação de valores na educação superior, a educação em todos os níveis de ensino e a tomada de decisões sobre as decisões corretas em circunstâncias conflitantes, cada dia a sociedade é uma questão de competência, a capacidade de segregar a crise de valores Que viven os profesionales universitários, são de importância vital que os docentes estipularam são o enfoque de valores, porque tanto a misión que todo o docente debe tener es convertir no modelo do alumso para que este aprenda um imitarlo, pues es esta la manera Mais eficaz de enseñar valores a los alumnos, por otra parte los docentes deben tener claro que no solo están formando buenos profesionales desde o ponto de vista do desenvolvimento, o que é o mesmo que forma ser humano que impactar na solução dos problemas de uma sociedade . La educación en valores não solos depende de los profesores es un trabajo en conjunto con as autoridades universitarias, en el trabajoexunidad como la Universidad "Luis Vargas Torres" de Esmeraldas, Ecuador, hizo un estudio que tuvo como finalidad recoger sugerencias en cuanto a la A formação de valores das escolas de engenharia, por sua vez, as escolas de ensino básico estão implementando ferramentas que permitem detectar as debilidades em conjunto com os programas de formação universitária.

Palavras chave: Valores; universidad; docentes; formação universitária.

\section{Introducción}

La educación de valores es una exigencia clave para el siglo XXI como proceso que sirve para adaptarse a los imperativos del mundo en constantes cambios, transformaciones, particularmente en las universidades como expresión del desarrollo social, por lo que debe exigirse el derecho a ella y asumir el deber de integración para su cuidado. (González, 2016)

Los principios y valores son necesarios para el desarrollo de nuestra vida, los seres humanos vivimos diferentes procesos a lo largo del tiempo y uno de ellos es la formación universitaria.

Sabemos que la educación es valores y los valores que hay que resaltar en la educación superior garantizan el futuro en la nación, Los educadores son los amigos del pueblo. Esta tarea educativa 
no es posible sin la cooperación de las instituciones, en este caso la universidad, por ello es imprescindible trabajar de la mano, acompañando el proceso de formación integral desde un proyecto de vida. (Molina, 2008)

La educación universitaria no sólo conlleva la enseñanza de conocimientos, competencias y habilidades dentro de una profesión o disciplina, también incluye la transmisión de hábitos, actitudes y valores con un contenido ético. (Barba \& Alcántara, 2003)

La formación de valores en la educación superior es un tema bastante complicado de abordar, por ello se debe contar con docentes que se preocupen por formar profesionales éticos, a fin de impactar y romper estructuras que perjudique el desarrollo de la sociedad.

No es secreto que la formación en valores se encuentra en crisis en muchas partes del mundo, se han formulado en varios países de América Latina un llamado de alerta al respecto. Algunos estudiosos consideran la formación en valores como el problema más importante de la educación contemporánea. Para esto han vinculado la formación de valores a cada programa de estudio de las diferentes especialidades a modo de implantarlos a la realidad social con la finalidad de buscar vínculos directos con la cotidianidad que nos permita demostrar al estudiantado lo que en realidad es éticamente válido para nuestras sociedades. (Viamontes, 2012)

Definitivamente si un país quiere desarrollar profesionales competitivos, que marquen la diferencia, que puedan realizar trabajos con sello de calidad, deberá establecer objetivos claros basados en la formación de valores, los cuales garantizaran una ética profesional que contribuirá al futuro de una nación.

\section{Método y materiales}

Esta investigación está enfocada en el estudio de la Formación de Valores en la Educación Superior. Por tanto, se trata de una revisión bibliográfica vigente y de toda documentación importante al respecto.

La revisión se ha centrado en textos, documentos y artículos científicos publicados disponibles en la web, considerando que aquella herencia de la globalización nos permite acceder a mayor y mejor información a través de las herramientas tecnológicas. 
El motor de búsqueda ha sido herramientas académicas de la web que direccionan específicamente a archivos con validez y reconocimiento científico, descartando toda información no confirmada o sin las respectivas referencias bibliográficas.

\section{Resultados}

Los Países Latinoamericanos están innovando en el desarrollo de nuevas estrategias que permita formar a los profesionales desde la educación de los valores.

Investigaciones realizadas en la Universidad Católica de Salta, Argentina Nelson E. J. (2008) demuestran que a partir de un diagnóstico en las carreras de Ingeniería donde se observa que no se atiende la formación humanista aun cuando en los estatutos de la referida universidad se declara la necesidad de la formación integral del profesional. En un estudio exploratorio realizado en la carrera de Ingeniería Civil a partir de encuestas realizadas a docentes y estudiantes se pudo constatar que para los docentes la formación en valores es algo importante y necesario, entre los valores más importantes a desarrollar en los futuros ingenieros destacan: la honestidad, el respeto y la solidaridad. Sin embargo, al preguntarles cómo trabajan estos valores en la carrera el (51\%) responde con charlas surgidas en clases o encuentros informales con los alumnos mientras que solo un $7 \%$ responde de manera directa y sistemática, exponiendo el tema y exigiendo calidad en las tareas. (Dubravcic, 2015)

Resultados semejantes se encuentran en otra Investigación realizada en carreras de Ingeniería en la Universidad del País Vasco, Usategui E. (2010). Estos autores refieren que en el campo de la Ingeniería el debate en torno a la importancia de la formación humanista del profesional no era algo central. En encuestas aplicadas a profesores de las carreras de Ingeniería y a egresados de la referida universidad, se observa que hay una escasa preocupación por el problema de la formación en valores. Al respecto los autores señalan:

El profesorado en general no es consciente de que también a través de los contenidos técnicos, implícitamente se introduzcan valores y de su papel en esa Inculcación. Incluso entre quienes advierten la conveniencia de que, entre los límites impuestos por las diferencias entre los contenidos curriculares, se contemplen más cuestiones que las puramente técnicas o científicas y más objetivo que los puramente instrumentales, se observa que se habla de la Incorporación de los valores, la ética o la formación humanista, sobre todo como contenidos explícitos y auxiliares 
en nuevas asignaturas y actividades complementarias, lo que evidencia esa concepción disociada entre formación técnica y humana a la que venimos aludiendo. (Dubravcic, 2015)

En el 2015 la Universidad "Luis Vargas Torres” de Esmeraldas, Ecuador, realizó un estudio mediante encuestas, para conocer la formación de valores dentro de la Facultad de Ciencias y Tecnología, el tamaño de la muestra utilizada fue del 10\% tanto de los estudiantes de Ingeniería como profesores de los diferentes años.

A continuación, se presentan un resumen de las respuestas que se obtuvieron a través de las encuestas:

Tabla 1. Valoración de los estudiantes sobre las cualidades o valores que caracterizan a un buen profesional.

\begin{tabular}{llcccccc}
\multirow{2}{*}{$\mathbf{N}^{\circ}$} & Cualidad & \multicolumn{2}{c}{$\mathbf{1}^{\circ} \mathbf{y} \mathbf{2}^{\circ} \mathbf{A n ̃ o}$} & \multicolumn{2}{c}{$\mathbf{3}^{\circ} \mathbf{4}^{\mathbf{0}} \mathbf{y} \mathbf{5}^{\circ}$ Año } & \multicolumn{2}{c}{ Total } \\
\cline { 3 - 8 } & & $\mathbf{N}^{\circ}$ & $\%$ & $\mathbf{N}^{\circ}$ & $\%$ & $\mathbf{N}^{\circ}$ & $\%$ \\
1 & Puntualidad & 124 & 15,54 & 216 & 16.56 & 340 & 16,18 \\
2 & Honestidad & 88 & 11,03 & 127 & 9,74 & 215 & 10,23 \\
3 & Iniciativa & 88 & 11,03 & 126 & 9,66 & 214 & 10,18 \\
4 & Solidaridad & 40 & 5,01 & 70 & 5,37 & 110 & 5,23 \\
5 & Humanismo & 35 & 4,39 & 56 & 4,29 & 91 & 4,33 \\
6 & Trabajo en equipo & 84 & 10,53 & 132 & 10,12 & 216 & 10,28 \\
7 & Respeto & 88 & 11,03 & 167 & 12,81 & 255 & 12,13 \\
8 & Creatividad & 107 & 13,41 & 159 & 12,19 & 266 & 12,65 \\
9 & Responsabilidad & 139 & 17,42 & 241 & 18,48 & 380 & 18,08 \\
10 & Otro & 5 & 0,63 & 10 & 0,77 & 15 & 0,71 \\
& Total & 798 & 100 & 1.304 & 100 & 2.102 & 100 \\
\hline
\end{tabular}

Fuente: (Dubravcic, 2015).

En la Tabla 1. Se observa la valoración de los estudiantes sobre las cualidades o valores que caracterizan a un buen profesional, en donde el valor más importante para los estudiantes de $1^{\circ} \mathrm{y}$ $2^{\circ}$ año es la responsabilidad y para los de $3^{\circ} 4^{\circ}$ y $5^{\circ}$ además dela responsabilidad consideran la puntualidad. Efectivamente, estos dos valores marcan la diferencia en lo que es ser un buen profesional, la puntualidad es sinónimo de cortesía, educación y respeto, por otra parte, la responsabilidad representa seriedad y compromiso. Si el mundo estuviese lleno de profesionales con estos valores el impacto en la sociedad sería una solución a los problemas. 
Tabla 2. Fortalezas que perciben los estudiantes en la formación de valores en su carrera.

\begin{tabular}{|c|c|c|c|c|c|c|c|}
\hline \multirow[t]{2}{*}{$\mathbf{N}^{\circ}$} & \multirow[t]{2}{*}{ Cualidad } & \multicolumn{2}{|c|}{$1^{\circ}$ y $2^{\circ}$ Año } & \multicolumn{2}{|c|}{$3^{\circ}, 4^{\circ}$ y $5^{\circ}$ Año } & \multicolumn{2}{|c|}{ Total } \\
\hline & & $\mathbf{N}^{\circ}$ & $\%$ & $\mathrm{~N}^{\circ}$ & $\%$ & $\mathbf{N}^{\circ}$ & $\%$ \\
\hline 1 & Puntualidad & 112 & 9,61 & 150 & 8,61 & 262 & 9,01 \\
\hline 2 & Honestidad & 67 & 5,75 & 69 & 3,96 & 136 & 4,68 \\
\hline 3 & Iniciativa & 76 & 6,52 & 96 & 5,51 & 172 & 5,91 \\
\hline 4 & Solidaridad & 50 & 4,29 & 79 & 4,53 & 129 & 4,44 \\
\hline 5 & Humanismo & 28 & 2,40 & 31 & 1,78 & 59 & 2,03 \\
\hline 6 & Trabajo en Equipo & 95 & 8,15 & 126 & 7,23 & 221 & 7,60 \\
\hline 7 & Respeto a unos & 81 & 6,95 & 121 & 6,94 & 202 & 6,95 \\
\hline 8 & Creatividad & 87 & 7,47 & 122 & 7,0 & 209 & 7,19 \\
\hline 9 & Respeto a otros & 43 & 3,69 & 51 & 2,93 & 94 & 3,23 \\
\hline 10 & Responsabilidad & 106 & 9,10 & 161 & 9,24 & 267 & 9,18 \\
\hline 11 & Otro & -- & -- & 1,0 & 0,06 & 1,0 & 0,03 \\
\hline & Total & 745 & 100 & 1.007 & 100 & 1.752 & 100 \\
\hline
\end{tabular}

Fuente: (Dubravcic, 2015)

En la Tabla 2 se puede evidenciar que las fortalezas que perciben los estudiantes del $1^{\circ}$ al $5^{\circ}$ año, en la formación de su carrera son la responsabilidad y puntualidad.

Tabla 3. Debilidades que perciben los estudiantes en la formación de valores en su carrera.

\begin{tabular}{clcccccc}
\multirow{2}{*}{$\mathbf{N}^{\circ}$} & Cualidad & \multicolumn{2}{c}{$\mathbf{1}^{\circ} \mathbf{y} \mathbf{2}^{\circ} \mathbf{A n ̃ o}$} & \multicolumn{3}{c}{$\mathbf{3}^{\circ} \mathbf{4}^{\circ} \mathbf{y} \mathbf{5}^{\circ} \mathbf{A n ̃ o}$} & \multicolumn{2}{c}{ Total } \\
\cline { 3 - 8 } & $\mathbf{N}^{\circ}$ & $\%$ & $\mathbf{N}^{\circ}$ & $\%$ & $\mathbf{N}^{\circ}$ & $\%$ \\
1 & No puntualidad & 70 & 6,01 & 123 & 7,06 & 193 & 6,64 \\
2 & No honestidad & 30 & 2,58 & 72 & 4,13 & 102 & 3,51 \\
3 & No iniciativa & 27 & 2,32 & 61 & 3,50 & 88 & 3,03 \\
4 & No solidaridad & 51 & 4,38 & 86 & 4,93 & 137 & 4,71 \\
5 & No humanismo & 62 & 5,32 & 71 & 4,07 & 133 & 4,57 \\
6 & No trabajo en equipo & 36 & 3,09 & 85 & 4,88 & 121 & 4,16 \\
7 & No respeto a uno & 34 & 2,92 & 71 & 4,07 & 105 & 3,61 \\
8 & No creatividad & 34 & 2,92 & 52 & 2,98 & 86 & 2,96 \\
9 & No respeto a otros & 27 & 2,32 & 25 & 1,43 & 52 & 1,79 \\
10 & No responsabilidad & 46 & 3,95 & 85 & 4,88 & 131 & 4,50 \\
11 & Otro & 3 & 0,26 & 5 & 0,29 & 8 & 0,28 \\
& Total & 420 & 100 & 736 & 100 & 1.156 & 100
\end{tabular}

Fuente: (Dubravcic, 2015).

En el caso de las debilidades Tabla 3 que fueron mencionadas sobresale claramente la impuntualidad, que fue mencionado en mayor porcentaje por los estudiantes. La opinión de los docentes, fue recabada a través de encuestas que se aplicaron en un clima favorable por la buena predisposición de los docentes con la finalidad de mejorar la calidad de ingenieros que son 
formados año tras año en la Facultad de Ciencias y Tecnología de la Universidad "Luis Vargas Torres" de Esmeraldas. La encuesta, tuvo como finalidad recoger sugerencias en cuanto a la formación de valores de las Carreras de Ingeniería.

Tabla 4. Opinión de los docentes sobre los valores que deben caracterizar el desempeño profesional del ingeniero

\begin{tabular}{llcc}
$\mathbf{N}^{\circ}$ & \multicolumn{1}{c}{ Respuestas } & Frecuencia & $\%$ \\
\hline 1 & Conocimiento técnico-práctico & 7 & 1,46 \\
2 & Curiosidad para investigar, perseverancia & 7 & 1,46 \\
3 & Honestidad y transparencia & 12 & 2,51 \\
4 & Visión realista & 15 & 3,14 \\
5 & Competente & 19 & 3,97 \\
6 & Iniciativa e imaginación & 26 & 5,44 \\
7 & Responsabilidad & 26 & 5,44 \\
8 & Autosuperación y preparación & 27 & 5,65 \\
9 & Puntualidad & 28 & 5,86 \\
10 & Equidad y solidaridad & 35 & 7,32 \\
11 & Compromiso & 46 & 9,62 \\
12 & Ética profesional & 55 & 11,51 \\
13 & Respeto & 55 & 11,51 \\
14 & Moral, capacidad de trabajo en grupo & 55 & 11,51 \\
15 & Incorruptibilidad & 65 & 13,60 \\
& Total & 478 & 100 \\
\hline
\end{tabular}

Fuente: (Dubravcic, 2015)

Con respecto a los valores morales Tabla 4, que deben tener los Ingenieros en el desempeño profesional, los docentes consideran que no deben ser "corruptos", para lo cual, deber tener "ética profesional, respeto, moral y capacidad para trabajar en grupo", aspectos que se relaciones con las cualidades que fueron mencionados en la Tabla 4, donde sobresale la responsabilidad y el compromiso con la sociedad que de tener los profesionales ingenieros.

De acuerdo a los resultados obtenidos en esta encuesta, se ve claramente la ausencia de formación de valores en los estudiantes de la carrera de Ingeniería, según (Dubravcic, 2015) es necesario capacitar los docentes en la educación de valores.

\section{Discusión y conclusiones}

La educación superior en ningún momento debe descuidar la formación de valores en los estudiantes, pues el futuro de una nación está en mano de los profesionales. 
En los últimos años en Latinoamérica ha habido un deterioro elevado en los valores de los estudiantes universitarios, algunos autores opinan que esto se debe a la falta de inversión, otros dicen que se debe al desperdicio del dinero por no contar con planes rigurosos y enfocados.

Es indiscutible que la universidad es un espacio importante de socialización, pero a veces toma direcciones no deseables, ya que las autoridades están más enfocadas en el trabajo administrativo que lo referente a los valores morales éticos; ante este panorama que se presenta es necesario definir acciones que protejan la identidad de los universitarios y educar en correspondencia con los valores que sustentan la sociedad en que viven; atendiendo esta necesidad de cómo formar en valores éticos y morales deberá ser través proyectos que fortalezcan el desarrollo social, a través de los valores para adquirir consciencia de su quehacer universitario y asumir su identidad como alumno universitario. (Montesinos, 2010)

Recalcar que la ética profesional sobre todo en conjunto con los demás valores, formen la importancia de resaltar y comprender las inquietudes que existen entre los estudiantes sobre cualquier tema en general, el alumno pueda tomar decisiones en el rol de transmisor-receptor de valores para considerarlos en cuestiones académicas y que el sistema pedagógico de las universidades sea flexible con el fin de que el estudiante las desarrolle plenamente en su formación integral como parte de su educación superior.

Es sumamente significativo que en los estudiantes con el apoyo de sus profesores se esfuercen en aplicar los valores en su vida cotidiana y profesional y así cumplir con los distintos ejes en los que puede llevarse a cabo su formación superior, los jóvenes universitarios son el presente del país, por lo cual, se les debe inculcar los valores que forman parte de su vida y carrera, y al mismo tiempo los utilicen como herramientas que sirvan para lograr una mejor conexión que impacte en la sociedad del conocimiento, donde se desarrollarán como distinguidos profesionistas que sean personas de bien, además de solucionar problemas, que sean solidarios a sus principios y valores dentro de la sociedad. (Chapa, 2015)

\section{Referencias Bibliográficas}

Barba, M. L., \& Alcántara, S. A. (2003). Los valores y la formación universitaria. Revistas Científicas de América Latina y el Caribe, España y Portugal. 
Chapa, A. P. (2015). Valores Universitarios en los Jóvenes Estudiantes de la Facultad de Ciencias Políticas y Administración Pública, UANL. Ride.

Dubravcic, A. A. (2015). La enseñanza de valores en las carreras de Ingeniería de la Universidad Autónoma Juan Misael Saracho. Revistas Bolivianas.

González, $\quad$ R. $\quad$ G. (2016). Scielo. Obtenido de http://scielo.sld.cu/scielo.php?script=sci_arttext\&pid=S1727-81202016000100011

Molina, P. $\quad$ S. $\quad$ (2008). Scielo. Obtenido de http://www.scielo.org.ve/scielo.php?script=sci_arttext\&pid=S0001-63652008000100009

Montesinos, S. E. (2010). Gestiopolis. Obtenido de https://www.gestiopolis.com/los-valores-enla-formacion-de-los-universitarios-desde-la-construccion-de-su-indentidad/

Torres, A. R. (2005). Los Valores y las valoraciones en la educación. Mexico: Trillas.

Viamontes, O. (03 de 12 de 2012). Gestiopolis. Obtenido de https://www.gestiopolis.com/laformacion-valores-educacion-superior/ 